\title{
Error estimates for semi-discrete and fully discrete Galerkin finite element approximations of the general linear second order hyperbolic equation
}

\author{
M. Basson* $\quad$ B. Stapelberg ${ }^{\dagger} \quad \& \quad$ N. F. J. van Rensburg $\ddagger$ \\ Department of Mathematics and Applied Mathematics, \\ University of Pretoria, South Africa
}

\begin{abstract}
In this paper we derive error estimates for the semi-discrete and fully discrete Galerkin approximations of a general linear second order hyperbolic partial differential equation with general damping (which includes boundary damping). The results can be applied to a variety of cases e.g. vibrating systems of linked elastic bodies. The results generalize pioneering work of Dupont and complement a recent article by Basson and Van Rensburg.
\end{abstract}

Keywords: Damped vibration; Error estimates; Finite elements; Galerkin approximation; Second order hyperbolic equation.

\section{Introduction}

The vibration of elastic bodies and structures consisting of elastic bodies is a subject of considerable interest. Various linear and nonlinear models

${ }^{*}$ Email: madelein.basson@up.ac.za

${ }^{\dagger}$ Corresponding author. Email: belinda.stapelberg@up.ac.za

‡Email: nic.vanrensburg@up.ac.za 
for beams, plates and linked structures consisting of beams and plates are available in the literature (see e.g. the references in Section 7). An important consideration is the influence of damping on the properties of the solutions as well as the numerical approximations of these solutions.

A general linear second order hyperbolic equation was introduced in [1] which includes different types of damping. (The general problem is formulated in Section 2 of this article.) This includes the general second order wave equation but also vibrating beams, plates etc., since the variational forms are the same as for the wave equation. Even systems of linked elastic bodies can be accommodated. The usual spaces $\mathcal{L}^{2}(\Omega)$ and $H_{0}^{1}(\Omega)$ are replaced by general Hilbert spaces $W$ and $V$, where $V$ is a subset of $W$. In the general problem the $\mathcal{L}^{2}$-norm is replaced by the general inertia norm $\|\cdot\|_{W}$ and the well known energy norm for the wave equation (equivalent to the $H^{1}$ norm) is replaced by a general energy norm $\|\cdot\|_{V}$. Naturally certain properties of the spaces and their norms need to be assumed in a general setting (see Section 2).

The finite element method for the general problem is considered in [2], where it is assumed that the damping is weak or of viscous type. However, the theory can not be applied to boundary damping for example. Under rather restrictive assumptions error estimates for the general problem which includes modal damping were derived in the technical report [3] and $\mathrm{PhD}$ thesis [4], both unpublished. In [5] error estimates for the wave equation are derived for the undamped case as well as a problem with general boundary conditions. In this article the restriction of weak damping in [2] is lifted but at the expense of other assumptions. For the semi-discrete error estimate, we generalize the results in [5] and for the fully discrete error estimate we adapt the theory in [2] (a generalization of [6]).

An existence result is presented in Section 2 in variational form. This is more convenient for the finite element method as one can compare conditions for existence to conditions for convergence. We see that sufficient conditions for existence are also used to prove convergence but for the convergence results more regularity of solutions is assumed.

It is well known that the smoothness of a solution is important in convergence analysis. In this article attention is given to the differentiability of the projection of the exact solution, to identify where regularity is used in the analysis. It is possible that the exact solution does not have a second order derivative with respect to the energy norm but its projection may have a second order derivative (see Section 3). 
In $[5,6,7,8]$ and other publications error estimates are derived for the semi-discrete approximation and then for the fully discrete approximation without using the results already obtained. To do this, the assumption is made that the energy norm of the third or fourth order derivative of the exact solution exists. This assumption is rather restrictive and we avoid it by following the method in [2]. First an error estimate for the semidiscrete approximation with respect to the energy norm is derived. Then an estimate for the error in approximating the solution of the semi-discrete problem by the fully discrete approximation with respect to the inertia norm is derived. The final estimate follows trivially by the triangle inequality. Another advantage of this approach is that the convergence analysis for the fully discrete approximation is simplified.

The outline of the paper is as follows. In Section 3 the Galerkin finite element approximation for the general problem is formulated and in Section 4 error estimates for the semi-discrete approximation in the energy norm are presented. An unconditionally stable fully discrete scheme due to [6] is formulated in Section 5, with error estimates for the fully discrete approximation appearing in Section 6. Finally, the importance of the general theory in applications is discussed in Section 7.

\section{Existence and uniqueness of solutions}

In this section we consider the general linear hyperbolic problem, also referred to as the general linear vibration problem. Let $V, W$ and $X$ be Hilbert spaces such that $V \subset W \subset X$ with the inner products and norms as given below:

$$
\begin{aligned}
& X \text { has inner product }(\cdot, \cdot)_{X} \text { and norm }\|\cdot\|_{X} ; \\
& W \text { has inner product } c(\cdot, \cdot) \text { and norm }\|\cdot\|_{W} ; \\
& V \text { has inner product } b(\cdot, \cdot) \text { and norm }\|\cdot\|_{V} \text {. }
\end{aligned}
$$

Let $J$ be a bounded or unbounded interval, either an open interval containing zero or of the form $[0, T)$ or $[0, \infty)$. Let $Y$ be any Hilbert space and consider a function $u$ on $J$ with values in $Y$.

We write $u^{\prime}(t) \in Y$ if the derivative exists with respect to the norm of $Y$; 


$$
\begin{aligned}
& u \in C^{k}(J ; Y) \text { if } u^{(k)} \in C(J ; Y) . \\
& u^{(k)} \in \mathcal{L}^{2}(J ; Y) \text { if } u^{(k)}(t) \in Y \text { for each } t \text { and } \int_{J}\left\|u^{(k)}\right\|_{Y}^{2}<\infty
\end{aligned}
$$

Also, let $a$ be a bilinear form defined on $V$ and consider the following general problem, referred to as the general second order hyperbolic equation.

\section{Problem G}

Given a function $f:[0, T] \rightarrow X$, find a function $u \in C([0, T) ; V)$ such that $u^{\prime}$ is continuous at 0 and for each $t \in(0, T), u(t) \in V, u^{\prime}(t) \in V, u^{\prime \prime}(t) \in W$ and

$$
\begin{gathered}
c\left(u^{\prime \prime}(t), v\right)+a\left(u^{\prime}(t), v\right)+b(u(t), v)=(f(t), v)_{X} \quad \text { for each } v \in V, \\
\text { while } u(0)=u_{0} \text { and } \quad u^{\prime}(0)=u_{1} .
\end{gathered}
$$

The following assumptions are made for the existence results as well as the convergence theory.

E1 $V$ is dense in $W$ and $W$ is dense in $X$.

E2 There exists a positive constant $\kappa_{1}$ such that $\|v\|_{W} \leq \kappa_{1}\|v\|_{V}$ for each $v \in V$.

E3 There exists a positive constant $\kappa_{2}$ such that $\|w\|_{X} \leq \kappa_{2}\|w\|_{W}$ for each $w \in W$.

E4 The bilinear form $a$ is non-negative, symmetric and bounded on $V$, i.e. there exists a positive constant $K_{a}$ such that for $v, w \in V$,

$$
|a(u, v)| \leq K_{a}\|u\|_{V}\|v\|_{V} .
$$

Note that Assumption E4 differs from the corresponding assumption in [2] and this difference impacts on the existence and convergence theory, as is evident from the relevant sections. The following existence theorem is from [1].

Theorem 2.1. Suppose Assumptions E1, E2, E3 and $\boldsymbol{E}_{4}$ hold. If $u_{0} \in V$ and $u_{1} \in W$, and there exists some $y \in W$ such that

$$
b\left(u_{0}, v\right)+a\left(u_{1}, v\right)=c(y, v) \quad \text { for each } v \in V,
$$

then for each $f \in C^{1}([0, T] ; X)$ there exists a unique solution

$$
u \in C([0, T) ; V) \cap C^{1}([0, T), W) \cap C^{1}((0, T) ; V) \cap C^{2}((0, T) ; W)
$$

for Problem G. If $f=0$ then $u \in C^{1}([0, \infty) ; V) \cap C^{2}([0, \infty) ; W)$. 
Problem $\mathrm{G}$ is equivalent to a first order differential equation in the product space $H=V \times W$. For $x \in H$ we write $x=\left\langle x_{1}, x_{2}\right\rangle$ with $x_{1} \in V$ and $x_{2} \in W$. To reformulate Problem $\mathrm{G}$, a linear operator $A$ is constructed using the three bilinear forms $c, b$ and $a$. The domain $\mathcal{D}(A) \subset V \times W$ and $\left\langle u_{0}, u_{1}\right\rangle \in \mathcal{D}(A)$ if and only if $u_{0}$ and $u_{1}$ are in $V$ and there exists a $y \in W$ such that condition (2.2) holds. Problem G is equivalent to a problem of the form

$$
w^{\prime}=A w+g \text { with } w(0)=\left\langle u_{0}, u_{1}\right\rangle
$$

Due to Assumptions E1, E2 and E4, $A$ is the infinitesimal generator of a $C_{0}$ semigroup of contractions. Existence follows from semigroup theory (using Assumption E3).

Other existence results are available in the literature, for example [9] and [10]. The result in [1] is convenient for this article, since the result is given in terms of bilinear forms.

\section{Galerkin approximation}

Suppose $S^{h}$ is a finite dimensional subspace of $V$. We can now formulate the semi-discrete problem for our general linear vibration problem, Problem G. The Galerkin finite element approximation of Problem $G$ is referred to as Problem $\mathrm{G}^{h}$.

\section{Problem $G^{h}$}

Given a function $f:[0, T] \rightarrow X$, find a function $u_{h} \in C^{2}[0, T]$ such that for each $t \in(0, T)$

$$
\begin{gathered}
c\left(u_{h}^{\prime \prime}(t), v\right)+a\left(u_{h}^{\prime}(t), v\right)+b\left(u_{h}(t), v\right)=(f(t), v)_{X} \quad \text { for each } v \in S^{h} \\
\text { while } u_{h}(0)=u_{0}^{h} \text { and } \quad u_{h}^{\prime}(0)=u_{1}^{h} .
\end{gathered}
$$

The initial values $u_{0}^{h}$ and $u_{1}^{h}$ are elements of $S^{h}$ and must be chosen appropriately. See Subsection 4.2.

Existence of a solution to Problem $\mathrm{G}^{h}$ is no problem if $f$ is continuous, since $c$ is an inner product and $S^{h}$ is finite dimensional, (see Section 5). 


\subsection{Projection}

To obtain an estimate for the error $u(t)-u_{h}(t)$, a projection is used. The projection operator $P_{h}$ is defined by

$$
b\left(u-P_{h} u, v\right)=0 \text { for all } v \in S^{h} .
$$

We write $P$ for $P_{h}$ if no confusion is possible. We also use $P$ to denote the projection $P u$ of a function $u$, that is, $(P u)(t)=P u(t)$ for each $t \in(0, T)$. The projection is used to split the error $e_{h}(t)=u(t)-u_{h}(t)$ as follows: $e(t)=P u(t)-u_{h}(t)$ and $e_{p}(t)=u(t)-P u(t)$, i.e.

$$
e_{h}(t)=e(t)+e_{p}(t) .
$$

Estimates for the norm of $e_{p}$ can be obtained from approximation theory, see Subsection 3.2. It remains to find an estimate for $e(t)$.

It is well known that if $u \in C^{k}(J ; V)$ for $k=1,2$, then $P u \in C^{k}(J)$ and $(P u)^{(k)}=P u^{(k)}$ (see e.g. [2]). If $u$ is a solution of Problem G, then $P u \in$ $C^{1}(0, T)$. However, to prove Lemma 3.1 below the existence of $(P u)^{\prime \prime}$ is required.

\section{Assumption A1}

The solution $u$ of Problem $\mathrm{G}$ has the property that $(P u) \in C^{2}(0, T)$.

\section{Assumption A2}

The solution $u$ of Problem $G$ satisfies $u \in C^{1}([0, T) ; V) \cap C^{2}((0, T) ; V)$.

For the next three results (Proposition 3.1, Lemma 3.1 and Lemma 4.1), only Assumption A1 is required.

Proposition 3.1. If $u \in C^{2}((0, T) ; W)$ and $u$ satisfies Assumption A1, then

$$
e_{p} \in C^{2}((0, T) ; W) \text {. }
$$

Proof. Recall that $e_{p}(t)=u(t)-(P u)(t)$. Since norms in $S^{h}$ are equivalent, $P u \in C^{2}((0, T) ; W)$.

Lemma 3.1. If the solution $u$ of Problem G satisfies Assumption A1, then for all $v \in S^{h}$ and $t \in(0, T)$,

$$
c\left(e_{p}^{\prime \prime}(t), v\right)+a\left(e_{p}^{\prime}(t), v\right)+c\left(e^{\prime \prime}(t), v\right)+a\left(e^{\prime}(t), v\right)+b(e(t), v)=0 .
$$

Proof. The result follows from Proposition 3.1 and the fact that $P u \in C^{1}(0, T)$. 


\subsection{Projection error}

With few exceptions, partial differential equations for scalar or vector valued functions on a single domain are considered in the literature. For a system of linked elastic bodies more than one domain is encountered. It is necessary to generalize the approach in the literature for systems. (See Section 7 for an interpretation.)

Definition Generalized interpolation operator.

$\Pi u=\sum_{k=1}^{n} \phi_{k}(u) w_{k}$ where $\left\{w_{k}\right\}$ is a basis for $S^{h}$ and $\phi_{k}$ are linear functionals.

To formulate an assumption regarding the error when an element of $V$ is approximated by an element of $S^{h}$, we suppose $h$ is a parameter related to the dimension $n$ of $S^{h}$ and $h \rightarrow 0$ as $n \rightarrow \infty$.

\section{Assumption A3}

There exists a subspace $H(V, k)$ of $V$, an interpolation operator $\Pi$ and positive constants $C_{\Pi}$ and $\alpha$ (depending on $V$ and $k$ ) such that for $u \in H(V, k)$ :

$$
\|u-\Pi u\|_{V} \leq C_{\Pi} h^{\alpha}\|u\|_{H(V, k)}
$$

where $\|\cdot\|_{H(V, k)}$ is a norm or semi-norm associated with $H(V, k)$.

The following trivial consequence will be used in the general theory.

Proposition 3.2. There exists a subspace $H(V, k)$ of $V$ and positive constants $C_{\Pi}$ and $\alpha$ (depending on $V$ and $k$ ) such that for $u \in H(V, k)$ :

$$
\|u-P u\|_{V} \leq C_{\Pi} h^{\alpha}\|u\|_{H(V, k)}
$$

where $\|\cdot\|_{H(V, k)}$ is a norm or semi-norm associated with $H(V, k)$. 


\section{Error estimates for the semi-discrete ap- proximation}

\subsection{Fundamental estimate}

The following "energy" expression for $e$ is useful:

$$
\begin{aligned}
E(t) & =\frac{1}{2} c\left(e^{\prime}(t), e^{\prime}(t)\right)+\frac{1}{2} b(e(t), e(t)) \\
& =\frac{1}{2}\left\|e^{\prime}(t)\right\|_{W}^{2}+\frac{1}{2}\|e(t)\|_{V}^{2} .
\end{aligned}
$$

Lemma 4.1. If the solution $u$ of Problem $G$ satisfies Assumption A1, then for any $t \in(0, T)$,

$$
E^{\prime}(t) \leq-c\left(e_{p}^{\prime \prime}(t), e^{\prime}(t)\right)-a\left(e_{p}^{\prime}(t), e^{\prime}(t)\right) .
$$

Proof. Since $u_{h} \in C^{2}[0, T]$ and $P u \in C^{2}(0, T)$, it follows that $e \in C^{2}(0, T)$. Also, since $P u$ and $u_{h}$ are in $S^{h}, e(t) \in S^{h}$, and it follows that $e^{\prime}(t) \in S^{h}$. Choose $v=e^{\prime}(t)$ in Lemma 3.1, then

$c\left(e_{p}^{\prime \prime}(t), e^{\prime}(t)\right)+c\left(e^{\prime \prime}(t), e^{\prime}(t)\right)+a\left(e_{p}^{\prime}(t), e^{\prime}(t)\right)+a\left(e^{\prime}(t), e^{\prime}(t)\right)+b\left(e(t), e^{\prime}(t)\right)=0$.

From the definition of the "energy" expression, and since $b$ and $c$ are inner products, we have

$$
E^{\prime}(t)=c\left(e^{\prime \prime}(t), e^{\prime}(t)\right)+b\left(e(t), e^{\prime}(t)\right) .
$$

Combining this with (4.3) yields

$$
E^{\prime}(t)=-c\left(e_{p}^{\prime \prime}(t), e^{\prime}(t)\right)-a\left(e_{p}^{\prime}(t), e^{\prime}(t)\right)-a\left(e^{\prime}(t), e^{\prime}(t)\right) .
$$

Since $a\left(e^{\prime}(t), e^{\prime}(t)\right) \geq 0$, the result follows.

From (4.2) the aim is to obtain an estimate for $E(t)$ in terms of projection errors. Since $\left\|e^{\prime}(t)\right\|_{W}^{2}+\|e(t)\|_{V}^{2}=2 E(t)$, it will yield an estimate for $e(t)$.

There is a problem with the last term in (4.2) since

$$
a\left(e_{p}^{\prime}(t), e^{\prime}(t)\right) \leq K_{a}\left\|e_{p}^{\prime}(t)\right\|_{V}\left\|e^{\prime}(t)\right\|_{V},
$$

and $\left\|e^{\prime}(t)\right\|_{V}$ is not bounded by $E(t)$. Lemma 4.2 below enables us to estimate this term. (The idea is from [5].) We need the following auxiliary result. 
Proposition 4.1. If $e_{p} \in C^{1}([0, T) ; V) \cap C^{2}((0, T) ; V)$, then for any $t \in(0, T)$,

$$
\int_{0}^{t} a\left(e_{p}^{\prime}(\cdot), e^{\prime}(\cdot)\right)=a\left(e_{p}^{\prime}(t), e(t)\right)-a\left(e_{p}^{\prime}(0), e(0)\right)-\int_{0}^{t} a\left(e_{p}^{\prime \prime}(\cdot), e(\cdot)\right) .
$$

Proof. If $\left\{\phi_{1}, \phi_{2}, \ldots, \phi_{n}\right\}$ is a basis for $S^{h}$, then

$$
e(t)=\sum_{i=1}^{n} e_{i}(t) \phi_{i}
$$

and hence

$$
a\left(e_{p}^{\prime}, e\right)=\sum_{i=1}^{n} e_{i} a\left(e_{p}^{\prime}, \phi_{i}\right)
$$

As $e \in C^{1}(0, T)$, it follows that $e_{i} \in C^{1}(0, T)$ for $i=1,2, \ldots, n$ and

$$
a\left(e_{p}^{\prime}, e^{\prime}\right)=\sum_{i=1}^{n} e_{i}^{\prime} a\left(e_{p}^{\prime}, \phi_{i}\right) .
$$

Since $e_{p} \in C^{2}((0, T) ; V)$, for any $t \in(0, T)$,

$$
\begin{array}{r}
\left|(\delta t)^{-1}\left(a\left(e_{p}^{\prime}(t+h), \phi_{i}\right)-a\left(e_{p}^{\prime}(t), \phi_{i}\right)\right)-a\left(e_{p}^{\prime \prime}(t), \phi_{i}\right)\right| \\
\leq K_{a}\left\|(\delta t)^{-1}\left(e_{p}^{\prime}(t+h)-e_{p}^{\prime}(t)\right)-e_{p}^{\prime \prime}(t)\right\|_{V}\left\|\phi_{i}\right\|_{V}
\end{array}
$$

and it follows that, for $i=1,2, \ldots, n, a\left(e_{p}^{\prime}(\cdot), \phi_{i}\right)$ is differentiable on $(0, T)$ with

$$
\frac{d}{d t} a\left(e_{p}^{\prime}(t), \phi_{i}\right)=a\left(e_{p}^{\prime \prime}(t), \phi_{i}\right)
$$

Combining (4.4), (4.5) and (4.6) yields

$$
\frac{d}{d t} a\left(e_{p}^{\prime}(t), e(t)\right)=a\left(e_{p}^{\prime \prime}(t), e(t)\right)+a\left(e_{p}^{\prime}(t), e^{\prime}(t)\right)
$$

Now, integrate both sides and use the fundamental theorem of calculus. The result follows.

Remark. The assumption $e_{p} \in C^{1}([0, T) ; V) \cap C^{2}((0, T) ; V)$ in Proposition 4.1 is a consequence of Assumption A2. 
Lemma 4.2. If the solution $u$ of Problem $G$ satisfies Assumption A2, then for any $t \in(0, T)$

$$
\begin{gathered}
\|e(t)\|_{V}+\left\|e^{\prime}(t)\right\|_{W} \leq \sqrt{24 e^{3 t}} K_{T}, \quad \text { where } \\
K_{T}=\int_{0}^{T}\left\|e_{p}^{\prime \prime}(\cdot)\right\|_{W}+3 K_{a} \max \left\|e_{p}^{\prime}(t)\right\|_{V}+3 K_{a} \int_{0}^{T}\left\|e_{p}^{\prime \prime}(\cdot)\right\|_{V}+\left\|e^{\prime}(0)\right\|_{W} \\
+\sqrt{1+K_{a}}\|e(0)\|_{V}+\sqrt{K_{a}}\left\|e_{p}^{\prime}(0)\right\|_{V}
\end{gathered}
$$

Proof. We have the following estimates from Cauchy's inequality, Young's inequality and Equation (4.1)

$$
\begin{aligned}
\left|c\left(e_{p}^{\prime \prime}(t), e^{\prime}(t)\right)\right| & \leq\left\|e_{p}^{\prime \prime}(t)\right\|_{W}\left\|e^{\prime}(t)\right\|_{W} \leq \frac{1}{2}\left\|e_{p}^{\prime \prime}(t)\right\|_{W}^{2}+\frac{1}{2}\left\|e^{\prime}(t)\right\|_{W}^{2}, \\
\left\|e^{\prime}(t)\right\|_{W}^{2} & \leq 2 E(t) .
\end{aligned}
$$

Using the above estimates in Equation (4.2) and integrating on both sides yields

$$
E(t) \leq E(0)+\frac{1}{2} \int_{0}^{t}\left\|e_{p}^{\prime \prime}(\cdot)\right\|_{W}^{2}+\int_{0}^{t} E(\cdot)-\int_{0}^{t} a\left(e_{p}^{\prime}(\cdot), e^{\prime}(\cdot)\right) .
$$

Using Proposition 4.1 we obtain

$$
\begin{aligned}
E(t) \leq & E(0)+\frac{1}{2} \int_{0}^{t}\left\|e_{p}^{\prime \prime}(\cdot)\right\|_{W}^{2}+\int_{0}^{t} E(\cdot) \\
& -\left(a\left(e_{p}^{\prime}(t), e(t)\right)-a\left(e_{p}^{\prime}(0), e(0)\right)-\int_{0}^{t} a\left(e_{p}^{\prime \prime}(\cdot), e(\cdot)\right)\right) .
\end{aligned}
$$

We need estimates for the terms in brackets. We use Young's inequality and the definition of $E(t)$.

$$
\begin{aligned}
\left|a\left(e_{p}^{\prime}(t), e(t)\right)\right| & \leq K_{a}\left\|e_{p}^{\prime}(t)\right\|_{V}\|e(t)\|_{V} \\
& \leq K_{a} \varepsilon^{-2}\left\|e_{p}^{\prime}(t)\right\|_{V}^{2}+K_{a} \varepsilon^{2}\|e(t)\|_{V}^{2} \\
& \leq K_{a} \varepsilon^{-2}\left\|e_{p}^{\prime}(t)\right\|_{V}^{2}+2 K_{a} \varepsilon^{2} E(t),
\end{aligned}
$$

with $\varepsilon$ arbitrary. Also,

$$
\begin{aligned}
\left|\int_{0}^{t} a\left(e_{p}^{\prime \prime}(\cdot), e(\cdot)\right)\right| & \leq \int_{0}^{t}\left|a\left(e_{p}^{\prime \prime}(\cdot), e(\cdot)\right)\right| \\
& \leq K_{a} \varepsilon^{-2} \int_{0}^{t}\left\|e_{p}^{\prime \prime}(\cdot)\right\|_{V}^{2}+K_{a} \varepsilon^{2} \int_{0}^{t}\|e(\cdot)\|_{V}^{2} \\
& \leq K_{a} \varepsilon^{-2} \int_{0}^{t}\left\|e_{p}^{\prime \prime}(\cdot)\right\|_{V}^{2}+2 K_{a} \varepsilon^{2} \int_{0}^{t} E(\cdot) .
\end{aligned}
$$


Therefore we have that

$$
\begin{aligned}
E(t) \leq E(0)+ & \frac{1}{2} \int_{0}^{t}\left\|e_{p}^{\prime \prime}\right\|_{W}^{2}+\int_{0}^{t} E(\cdot)+2 K_{a} \varepsilon^{2} \int_{0}^{t} E(\cdot)+2 K_{a} \varepsilon^{2} E(t) \\
& +K_{a} \varepsilon^{-2}\left\|e_{p}^{\prime}(t)\right\|_{V}^{2}+a\left(e_{p}^{\prime}(0), e(0)\right)+K_{a} \varepsilon^{-2} \int_{0}^{t}\left\|e_{p}^{\prime \prime}(\cdot)\right\|_{V}^{2} \\
\leq & E(0)+\frac{1}{2} \int_{0}^{t}\left\|e_{p}^{\prime \prime}(\cdot)\right\|_{W}^{2}+\frac{3}{2} \int_{0}^{t} E(\cdot)+\frac{1}{2} E(t)+4 K_{a}^{2}\left\|e_{p}^{\prime}(t)\right\|_{V}^{2} \\
& +a\left(e_{p}^{\prime}(0), e(0)\right)+4 K_{a}^{2} \int_{0}^{t}\left\|e_{p}^{\prime \prime}(\cdot)\right\|_{V}^{2}
\end{aligned}
$$

by choosing $\varepsilon$ such that $2 K_{a} \varepsilon^{2}=\frac{1}{2}$.

Note that $\left|a\left(e_{p}^{\prime}(0), e(0)\right)\right| \leq \frac{1}{2} K_{a}\left\|e_{p}^{\prime}(0)\right\|_{V}^{2}+\frac{1}{2} K_{a}\|e(0)\|_{V}^{2}$. Rewriting (4.8) we get

$$
\begin{aligned}
\frac{1}{2} E(t) \leq & \frac{3}{2} \int_{0}^{t} E(\cdot)+\frac{1}{2} \int_{0}^{t}\left\|e_{p}^{\prime \prime}(\cdot)\right\|_{W}^{2}+4 K_{a}^{2}\left\|e_{p}^{\prime}(t)\right\|_{V}^{2} \\
& +4 K_{a}^{2} \int_{0}^{t}\left\|e_{p}^{\prime \prime}(\cdot)\right\|_{V}^{2}+E(0)+\frac{1}{2} K_{a}\left\|e_{p}^{\prime}(0)\right\|_{V}^{2}+\frac{1}{2} K_{a}\|e(0)\|_{V}^{2},
\end{aligned}
$$

which can be written in the form needed for the Gronwall inequality:

$$
\begin{aligned}
& E(t) \leq 3 \int_{0}^{t} E(\cdot)+K_{T}^{*}, \text { where the constant } \\
& \begin{aligned}
K_{T}^{*}= & \int_{0}^{T}\left\|e_{p}^{\prime \prime}(\cdot)\right\|_{W}^{2}+8 K_{a}^{2} \max \left\|e_{p}^{\prime}(t)\right\|_{V}^{2}+8 K_{a}^{2} \int_{0}^{T}\left\|e_{p}^{\prime \prime}(\cdot)\right\|_{V}^{2} \\
& \quad+\left\|e^{\prime}(0)\right\|_{W}^{2}+\left(1+K_{a}\right)\|e(0)\|_{V}^{2}+K_{a}\left\|e_{p}^{\prime}(0)\right\|_{V}^{2} .
\end{aligned}
\end{aligned}
$$

By applying the Gronwall inequality, we have $E(t) \leq e^{3 t} K_{T}^{*}$, and therefore

$$
\frac{1}{2}\left\|e^{\prime}(t)\right\|_{W}^{2}+\frac{1}{2}\|e(t)\|_{V}^{2} \leq e^{3 t} K_{T}^{*}
$$

Using the fact that $\|\cdot\|_{2}^{2} \leq n\|\cdot\|_{\infty}^{2} \leq n\|\cdot\|_{1}^{2}$, we obtain

$$
\begin{aligned}
K_{T}^{*} \leq 6 & {\left[\int_{0}^{T}\left\|e_{p}^{\prime \prime}(\cdot)\right\|_{W}+\sqrt{8} K_{a} \max \left\|e_{p}^{\prime}(t)\right\|_{V}+\sqrt{8} K_{a} \int_{0}^{T}\left\|e_{p}^{\prime \prime}(\cdot)\right\|_{V}\right.} \\
& \left.+\left\|e^{\prime}(0)\right\|_{W}+\sqrt{1+K_{a}}\|e(0)\|_{V}+\sqrt{K_{a}}\left\|e_{p}^{\prime}(0)\right\|_{V}\right]^{2} .
\end{aligned}
$$

The result then follows from the above inequality and the fact that $(a+b)^{2} \leq 2 a^{2}+2 b^{2}$. 


\subsection{Convergence and error estimates}

Lemma 4.3. Assume that the solution $u$ of Problem $G$ satisfies Assumption A2. Then, for any $t \in(0, T)$,

$$
\begin{gathered}
\left\|u(t)-u_{h}(t)\right\|_{V}+\left\|u^{\prime}(t)-u_{h}^{\prime}(t)\right\|_{W} \\
\leq\|u(t)-P u(t)\|_{V}+\left\|u^{\prime}(t)-P u^{\prime}(t)\right\|_{W}+\sqrt{24 e^{3 t}} K_{T}, \quad \text { where } \\
K_{T}=\int_{0}^{T}\left\|u^{\prime \prime}-P u^{\prime \prime}\right\|_{W}+3 K_{a} \max \left\|u^{\prime}(t)-P u^{\prime}(t)\right\|_{V}+3 K_{a} \int_{0}^{T}\left\|u^{\prime \prime}-P u^{\prime \prime}\right\|_{V} \\
+\left\|P u_{1}-u_{1}^{h}\right\|_{W}+\sqrt{1+K_{a}}\left\|P u_{0}-u_{0}^{h}\right\|_{V}+\sqrt{K_{a}}\left\|u_{1}-P u_{1}\right\|_{V} .
\end{gathered}
$$

Proof. The result follows directly from Lemma 4.2 .

Next we substitute the estimates for the projection errors.

Theorem 4.1. Suppose Assumption A3 holds for the space V. Assume also that the solution $u$ of Problem $G$ satisfies Assumption A2 and that $u^{\prime \prime} \in \mathcal{L}^{2}([0, T] ; H(V, k))$. Then, for any $t \in(0, T)$,

$$
\begin{aligned}
& \left\|u(t)-u_{h}(t)\right\|_{V}+\left\|u^{\prime}(t)-u_{h}^{\prime}(t)\right\|_{W} \\
& \leq C_{\Pi} h^{\alpha}\left(\|u(t)\|_{H(V, k)}+C_{b}\left\|u^{\prime}(t)\right\|_{H(V, k)}\right)+\sqrt{24 e^{3 t}} C_{\Pi} h^{\alpha}\left[\int_{0}^{T} C_{b}\left\|u^{\prime \prime}(\cdot)\right\|_{H(V, k)}\right. \\
& \left.\quad+3 K_{a} \max \left\|u^{\prime}(t)\right\|_{H(V, k)}+3 K_{a} \int_{0}^{T}\left\|u^{\prime \prime}(\cdot)\right\|_{H(V, k)}\right] \\
& \quad+\sqrt{24 e^{3 t}}\left[\left\|P u_{1}-u_{1}^{h}\right\|_{W}+\sqrt{1+K_{a}}\left\|P u_{0}-u_{0}^{h}\right\|_{V}+\sqrt{K_{a}}\left\|u_{1}-P u_{1}\right\|_{V}\right] .
\end{aligned}
$$

Proof. The result follows from Lemma 4.3, Assumption E2 and Proposition 3.2.

\section{Initial conditions}

The error depends on $u_{0}^{h}$ and $u_{1}^{h}$, but $u_{0}^{h}$ and $u_{1}^{h}$ are not given. In applications one must choose $u_{0}^{h}$ and $u_{1}^{h}$. If we choose $P u_{0}=u_{0}^{h}$, for example, then $\left\|P u_{0}-u_{0}^{h}\right\|_{V}=0$. In practice it is problematic. An obvious choice is to use the interpolants of $u_{0}$ and $u_{1}$.

Corollary 4.1. Suppose Assumption A3 holds for the space $V$ and $u_{0}$ and $u_{1}$ are in $H(V, k)$. Let $u_{0}^{h}=\Pi u_{0}$ and $u_{1}^{h}=\Pi u_{1}$. Assume also that the solution 
u of Problem $G$ satisfies Assumption A2 and $u^{\prime \prime} \in \mathcal{L}^{2}([0, T] ; H(V, k))$. Then,

$$
\begin{aligned}
& \left\|u(t)-u_{h}(t)\right\|_{V}+\left\|u^{\prime}(t)-u_{h}^{\prime}(t)\right\|_{W} \\
& \leq C_{\Pi} h^{\alpha}\left(\|u(t)\|_{H(V, k)}+C_{b}\left\|u^{\prime}(t)\right\|_{H(V, k)}\right)+\sqrt{24 e^{3 t}} C_{\Pi} h^{\alpha}\left[\int_{0}^{T} C_{b}\left\|u^{\prime \prime}(\cdot)\right\|_{H(V, k)}\right. \\
& \quad+3 K_{a} \max \left\|u^{\prime}(t)\right\|_{H(V, k)}+3 K_{a} \int_{0}^{T}\left\|u^{\prime \prime}(\cdot)\right\|_{H(V, k)} \\
& \left.\quad+2 C_{b}\left\|u_{1}\right\|_{H(V, k)}+2 \sqrt{1+K_{a}}\left\|u_{0}\right\|_{H(V, k)}+\sqrt{K_{a}}\left\|u_{1}\right\|_{H(V, k)}\right] .
\end{aligned}
$$

for $t \in(0, T)$.

Proof. The result follows from Theorem 4.1 and note that

$$
\begin{aligned}
\left\|P u_{0}-\Pi u_{0}\right\|_{V} & \leq\left\|P u_{0}-u_{0}\right\|_{V}+\left\|u_{0}-\Pi u_{0}\right\|_{V} \\
& \leq 2 C_{\Pi} h^{\alpha}\left\|u_{0}\right\|_{H(V, k)} ; \\
\left\|P u_{1}-\Pi u_{1}\right\|_{W} & \leq C_{b}\left\|P u_{1}-\Pi u_{1}\right\|_{V} \\
& \leq C_{b}\left\|P u_{1}-u_{1}\right\|_{V}+C_{b}\left\|u_{1}-\Pi u_{1}\right\|_{V} \\
& \leq 2 C_{b} C_{\Pi} h^{\alpha}\left\|u_{1}\right\|_{H(V, k)} .
\end{aligned}
$$

Remark. As mentioned in [2], it is sometimes possible to use the AubinNitche trick (see $[11,12]$ ) to obtain higher order estimates for the projection error. But the improved projection errors serve no purpose if the interpolants are used to approximate the initial values $u_{0}$ and $u_{1}$. If the starting values $u_{0}^{h}$ and $u_{1}^{h}$ are projections of $u_{0}$ and $u_{1}$ with respect to the inner product $c$ (as in [6]), then the result above can be improved.

\section{The fully discrete approximation}

It is well know that Problem $\mathrm{G}^{h}$ is equivalent to a system of second order ordinary differential equations (see e.g. [2]). Therefore, if $f \in C([0, T] ; X)$, then there exists a unique solution $u_{h} \in C^{2}[0, T]$ for Problem $G^{h}$ for each $u_{0}^{h}$ and $u_{1}^{h}$ in $S^{h}$. If $f \in C^{k}([0, T] ; X)$, then $u_{h} \in C^{2+k}[0, T]$ and if $f=0$ then $u_{h} \in C^{\infty}((-\infty, \infty))$. For more detail see [2]. 


\subsection{Baker's fully discrete Galerkin scheme}

A finite difference method is used to approximate the solution of the system in Problem $\mathrm{G}^{h}$. In [2] the authors follow [6] with an obvious modification to include the damping term. Note that Baker [6] compares the finite difference scheme to the exact solution $u$ of Problem G, whereas in [2] it is compared to the Galerkin approximation $u_{h}$ of Problem $\mathrm{G}^{h}$. To do the time discretization, suppose the interval $[0, T]$ is divided into $N$ steps of length $\tau=\frac{T}{N}$ and denote the approximation of $u_{h}\left(t_{k}\right)$ by $u_{k}^{h}$. Where no confusion is possible we write $u_{k}$ for $u_{k}^{h}$.

The difference between the solution of Problem G and the fully discrete approximation is:

$$
u\left(t_{k}\right)-u_{k}^{h}=\left[u\left(t_{k}\right)-u_{h}\left(t_{k}\right)\right]+\left[u_{h}\left(t_{k}\right)-u_{k}^{h}\right] .
$$

Following [2], we now derive an estimate for the error $u_{h}\left(t_{k}\right)-u_{k}^{h}$, and then use the triangle inequality to obtain a final estimate. This approach has two advantages. It is not necessary to assume the existence of a third or fourth order derivative for the exact solution and the convergence analysis for the fully discrete approximation is simplified. In the literature (see for example $[5,6,7,8,13,14,15]$ and [16]) error estimates are derived for the semi discrete approximation and then for the fully discrete approximation without using the results already obtained.

Notation For any sequence $\left\{x_{k}\right\} \subset R_{n}$ :

$$
\begin{gathered}
\delta_{t} x_{k}=\tau^{-1}\left[x_{k+1}-x_{k}\right], \\
x_{k+\frac{1}{2}}=\frac{1}{2}\left[x_{k+1}+x_{k}\right] .
\end{gathered}
$$

Following [6] and [2], consider the fully discrete problem in variational form. The vector $v_{k}$ in (5.1) below is an approximation for $u_{h}^{\prime}\left(t_{k}\right)$.

\section{Problem $G^{h}-\mathrm{D}$}

Find a sequence $\left\{u_{k}^{h}\right\} \subset S^{h}$ such that for $k=0,1,2, \ldots, N-1$,

$$
\begin{aligned}
\delta_{t} u_{k}^{h} & =v_{k+\frac{1}{2}}, \\
c\left(\delta_{t} v_{k}, \psi\right)+a\left(v_{k+\frac{1}{2}}, \psi\right)+b\left(u_{k+\frac{1}{2}}^{h}, \psi\right) & =\frac{1}{2}\left(\left[f\left(t_{k}\right)+f\left(t_{k+1}\right)\right], \psi\right)_{X}
\end{aligned}
$$

for each $\psi \in S^{h}$, while $u_{0}^{h}=u_{h}(0)$ and $v_{0}=u_{h}^{\prime}(0)$. 
Problem $\mathrm{G}^{h}$-D has a unique solution for any pair of vectors $u_{0}^{h}$ and $v_{0}$ in $S^{h}$ (see [6] or [2]).

\section{Remarks.}

1. The damping term $a(\cdot, \cdot)$ does not appear in [6] and in [2] it is bounded by $\|\cdot\|_{W}$. However, it is not bounded by $\|\cdot\|_{W}$ in the present case.

2. Other finite difference schemes were taken into consideration. In [14] the Newmark sheme (of which the central difference scheme is a special case) is used for the time discretization. The problem considered includes general damping. For the proof of the stability result the author refers to [15] where an undamped wave equation is considered. The article was studied in depth and we are convinced that the proof is not easy to adapt for general damping.

\section{Error estimates for the fully discrete ap- proximation}

\subsection{Stability}

The proof of the stability result is effectively the same as in [2]. However, for the sake of readability we present some of the main steps.

Consider the errors $e_{k}$ and $q_{k}$ :

$$
e_{k}=u_{h}\left(t_{k}\right)-u_{k} \quad \text { and } \quad q_{k}=v_{h}\left(t_{k}\right)-v_{k},
$$

where $v_{h}(t)=u_{h}^{\prime}(t)$. Note that $e_{0}=0$ and $q_{0}=0$.

Substitution of $t=t_{k}$ and $t=t_{k+1}$ into Problem $\mathrm{G}^{h}$ (Equation (3.1)) yields

$$
\begin{aligned}
& c\left(\tau^{-1}\left[v_{h}\left(t_{k+1}\right)-v_{h}\left(t_{k}\right)\right], \psi\right)+\frac{1}{2} a\left(v_{h}\left(t_{k+1}\right)+v_{h}\left(t_{k}\right), \psi\right) \\
& +\frac{1}{2} b\left(u_{h}\left(t_{k+1}\right)+u_{h}\left(t_{k}\right), \psi\right)=\frac{1}{2}\left(f\left(t_{k+1}\right)+f\left(t_{k}\right), \psi\right)_{X}+c\left(\rho_{k}, \psi\right),
\end{aligned}
$$

where

$$
\rho_{k}=\tau^{-1}\left[v_{h}\left(t_{k+1}\right)-v_{h}\left(t_{k}\right)\right]-\frac{1}{2}\left[v_{h}^{\prime}\left(t_{k+1}\right)+v_{h}^{\prime}\left(t_{k}\right)\right] .
$$


Next use

$$
\delta_{t} e_{k}=q_{k+\frac{1}{2}}+\sigma_{k}
$$

where

$$
\sigma_{k}=\tau^{-1}\left[u_{h}\left(t_{k+1}\right)-u_{h}\left(t_{k}\right)\right]-\frac{1}{2}\left[v_{h}\left(t_{k+1}\right)+v_{h}\left(t_{k}\right)\right] .
$$

It then follows that for any integer $n>0$,

$$
e_{n}=\tau \sum_{k=0}^{n-1} \delta_{t} e_{k}=\tau \sum_{k=0}^{n-1} q_{k+\frac{1}{2}}+\tau \sum_{k=0}^{n-1} \sigma_{k}
$$

Now let $s_{0}=0, s_{n}=\tau \sum_{k=0}^{n-1} e_{k+\frac{1}{2}}$, and

$$
\epsilon_{n}=\frac{\tau}{2} \rho_{n}+\tau \sum_{k=0}^{n-1} \rho_{k}+\sigma_{n} \quad \text { for } \quad n=1,2, \ldots N-1 .
$$

Let $\nu$ be an integer such that $2 \leq \nu \leq N$. From the equations above it is proved in [2] that

$$
\begin{aligned}
& \left\|e_{\nu}\right\|_{W}^{2}-\left\|e_{1}\right\|_{W}^{2}+\left\|s_{\nu}\right\|_{V}^{2}-\left\|s_{1}\right\|_{V}^{2} \\
& \quad \leq 4 T \tau \sum_{n=1}^{\nu-1}\left\|\epsilon_{n}\right\|_{W}^{2}+\frac{\tau}{4 T} \sum_{n=1}^{\nu-1}\left\|e_{n+\frac{1}{2}}\right\|_{W}^{2} .
\end{aligned}
$$

For $n=0$ it is also proved in [2] that

$$
\begin{aligned}
& \left\|e_{1}\right\|_{W}^{2}+\left\|s_{1}\right\|_{V}^{2}+\frac{\tau}{2} a\left(e_{1}, e_{1}\right) \\
& \quad=\frac{\tau^{2}}{2} c\left(\rho_{0}, e_{1}\right)+\tau c\left(\sigma_{0}, e_{1}\right)+\frac{\tau^{2}}{2} a\left(\sigma_{0}, e_{1}\right) .
\end{aligned}
$$

From the Cauchy-Schwartz and Young's inequalities, and since $a\left(e_{1}, e_{1}\right) \geq 0$, we find

$$
\begin{aligned}
\left\|e_{1}\right\|_{W}^{2}+\left\|s_{1}\right\|_{V}^{2} \leq & \frac{\tau^{2}}{2}\left\|\rho_{0}\right\|_{W}\left\|e_{1}\right\|_{W}+\tau\left\|\sigma_{0}\right\|_{W}\left\|e_{1}\right\|_{W}+\frac{\tau^{2}}{2} K_{a}\left\|\sigma_{0}\right\|_{V}\left\|e_{1}\right\|_{V} \\
\leq & \tau^{4}\left\|\rho_{0}\right\|_{W}^{2}+\frac{1}{16}\left\|e_{1}\right\|_{W}^{2}+4 \tau^{2}\left\|\sigma_{0}\right\|_{W}^{2}+\frac{1}{16}\left\|e_{1}\right\|_{W}^{2} \\
& \quad+\tau^{4} C_{a}^{2}\left\|\sigma_{0}\right\|_{V}^{2}+\frac{1}{16}\left\|e_{1}\right\|_{V}^{2}
\end{aligned}
$$


Now, combine (6.4) and (6.5) to obtain

$$
\begin{aligned}
\left\|e_{\nu}\right\|_{W}^{2} \leq & 4 T \tau \sum_{n=1}^{\nu-1}\left\|\epsilon_{n}\right\|_{W}^{2}+\frac{\tau}{4 T} \sum_{n=1}^{\nu-1}\left\|e_{n+\frac{1}{2}}\right\|_{W}^{2} \\
& +\frac{1}{16}\left(2\left\|e_{1}\right\|_{W}^{2}+\left\|e_{1}\right\|_{V}^{2}\right)+\tau^{4}\left\|\rho_{0}\right\|_{W}^{2}+4 \tau^{2}\left\|\sigma_{0}\right\|_{W}^{2}+\tau^{4} K_{a}^{2}\left\|\sigma_{0}\right\|_{V}^{2} \\
\leq & 4 T \tau \sum_{n=1}^{\nu-1}\left\|\epsilon_{n}\right\|_{W}^{2}+\frac{1}{2} \max \left\|e_{n}\right\|_{W}^{2}+\frac{1}{16}\left\|e_{1}\right\|_{V}^{2}+\tau^{4}\left\|\rho_{0}\right\|_{W}^{2} \\
& +4 \tau^{2}\left\|\sigma_{0}\right\|_{W}^{2}+\tau^{4} K_{a}^{2}\left\|\sigma_{0}\right\|_{V}^{2} .
\end{aligned}
$$

Due to the fact that the bilinear form $a$ represents general damping, Equation (6.5) and hence also (6.6), differ from the corresponding inequalities in [2]. The stability result follows from (6.6).

Lemma 6.1. Stability

$$
\begin{aligned}
\max \left\|e_{n}\right\|_{W}^{2} \leq & 8 T \tau \sum_{n=0}^{N-1}\left\|\epsilon_{n}\right\|_{W}^{2}+\frac{1}{8}\left\|e_{1}\right\|_{V}^{2}+2 \tau^{4}\left\|\rho_{0}\right\|_{W}^{2}+8 \tau^{2}\left\|\sigma_{0}\right\|_{W}^{2} \\
& +2 \tau^{4} K_{a}\left\|\sigma_{0}\right\|_{V}^{2}
\end{aligned}
$$

\subsection{Convergence}

Estimates for $\rho_{k}, \sigma_{k}$ and $e_{1}$ are required. The estimates for $\rho_{k}$ and $\sigma_{k}$ (with respect to $\|\cdot\|_{W}$ ) were derived (using Taylor expansions) in [2]:

$$
\left\|\rho_{k}\right\|_{W}^{2} \leq \tau^{4} \max \left\|v_{h}^{\prime \prime \prime}\right\|_{W}^{2}
$$

and

$$
\left\|\sigma_{k}\right\|_{W}^{2} \leq \tau^{4} \max \left\|u_{h}^{\prime \prime \prime}\right\|_{W}^{2}
$$

From the definition of $\epsilon_{n}$ it then follows that

$$
\left\|\epsilon_{n}\right\|_{W}^{2} \leq 5 T^{2} \tau^{4} \max \left\|v_{h}^{\prime \prime \prime}\right\|_{W}^{2}+4 \tau^{4} \max \left\|u_{h}^{\prime \prime \prime}\right\|_{W}^{2} .
$$

For general damping we also need estimates for $\left\|\sigma_{0}\right\|_{V}$ and $\left\|e_{1}\right\|_{V}$. The estimate for $\left\|\sigma_{0}\right\|_{V}$ is obtained by the same procedure as the Estimate (6.9). 
(An orthonormal basis in $V$ is used instead of an orthonormal basis in $W$.) It follows that

$$
\left\|\sigma_{k}\right\|_{V}^{2} \leq \tau^{4} \max \left\|u_{h}^{\prime \prime \prime}\right\|_{V}^{2}
$$

To estimate $e_{1}$, note that $e_{1}=\frac{\tau}{2} q_{1}+\tau \sigma_{0}$ (substituting $k=0$ in Equation (6.2)) and therefore

$$
\begin{aligned}
\left\|e_{1}\right\|_{V}^{2} & \leq\left(\left\|\frac{\tau}{2} q_{1}\right\|_{V}+\left\|\tau \sigma_{0}\right\|_{V}\right)^{2} \\
& =\left\|\frac{\tau}{2} q_{1}\right\|_{V}^{2}+\left\|\tau \sigma_{0}\right\|_{V}^{2}+2\left\|\frac{\tau}{2} q_{1}\right\|_{V}\left\|\tau \sigma_{0}\right\|_{V} \\
& \leq 2\left(\frac{\tau^{2}}{4}\left\|q_{1}\right\|_{V}^{2}+\tau^{2}\left\|\sigma_{0}\right\|_{V}^{2}\right) .
\end{aligned}
$$

Consequently an estimate for $\left\|q_{1}\right\|_{V}$ is required. First, from the definition of $\sigma_{k}$ we find that

$$
\begin{aligned}
& \frac{1}{2} b\left(u_{h}\left(t_{1}\right)+u_{h}\left(t_{0}\right), \psi\right)=\frac{\tau}{2} b\left(\tau^{-1}\left[u_{h}\left(t_{1}\right)-u_{h}\left(t_{0}\right)\right], \psi\right)+b\left(u_{h}\left(t_{0}\right), \psi\right) \\
& =\frac{\tau}{2} b\left(\frac{1}{2}\left[v_{h}\left(t_{1}\right)+v_{h}\left(t_{0}\right)\right]+\sigma_{0}, \psi\right)+b\left(u_{h}\left(t_{0}\right), \psi\right) \\
& =\frac{\tau}{4} b\left(v_{h}\left(t_{1}\right), \psi\right)+\frac{\tau}{4} b\left(v_{h}\left(t_{0}\right), \psi\right)+\frac{\tau}{2} b\left(\sigma_{0}, \psi\right)+b\left(u_{h}\left(t_{0}\right), \psi\right)
\end{aligned}
$$

Using Equation (6.11), Equation (6.1) (with $k=0$ ) can be rewritten as

$$
\begin{aligned}
& c\left(v_{h}\left(t_{1}\right), \psi\right)+\frac{\tau}{2} a\left(v_{h}\left(t_{1}\right), \psi\right)+\frac{\tau^{2}}{4} b\left(v_{h}\left(t_{1}\right), \psi\right) \\
& =c\left(v_{h}\left(t_{0}\right), \psi\right)-\frac{\tau}{2} a\left(v_{h}\left(t_{0}\right), \psi\right)-\frac{\tau^{2}}{4} b\left(v_{h}\left(t_{0}\right), \psi\right)-\tau b\left(u_{h}\left(t_{0}\right), \psi\right)-\frac{\tau^{2}}{4} b\left(\sigma_{0}, \psi\right) \\
& \quad+\tau c\left(\rho_{0}, \psi\right)+\frac{\tau}{2}\left(\left[f\left(t_{0}\right)+f\left(t_{1}\right)\right], \psi\right)_{X} .
\end{aligned}
$$

The following identity is from [2] (which is derived in the proof of existence of a unique solution to Problem $\left.\mathrm{G}^{h}-\mathrm{D}\right)$ :

$$
\begin{aligned}
\frac{\tau}{2} b\left(u_{1}+u_{0}, \psi\right) & =\frac{\tau^{2}}{2} b\left(\tau^{-1}\left[u_{1}-u_{0}\right], \psi\right)+\frac{\tau}{2} b\left(u_{0}, \psi\right)+\frac{\tau}{2} b\left(u_{0}, \psi\right) \\
& =\frac{\tau^{2}}{4} b\left(v_{1}, \psi\right)+\frac{\tau}{4} b\left(v_{0}, \psi\right)+\tau b\left(u_{0}, \psi\right) .
\end{aligned}
$$


Substituting Equation (6.13) into Equation (5.2) yields

$$
\begin{aligned}
& c\left(v_{1}, \psi\right)+\frac{\tau}{2} a\left(v_{1}, \psi\right)+\frac{\tau^{2}}{4} b\left(v_{1}, \psi\right) \\
& =c\left(v_{0}, \psi\right)-\frac{\tau}{2} a\left(v_{0}, \psi\right)-\frac{\tau^{2}}{4} b\left(v_{0}, \psi\right)-\tau b\left(u_{0}, \psi\right)+\frac{\tau}{2}\left(\left[f\left(t_{0}\right)+f\left(t_{1}\right)\right], \psi\right)_{X} .
\end{aligned}
$$

Subtracting Equation (6.14) from Equation (6.12) yields

$$
\begin{aligned}
& c\left(q_{1}, \psi\right)+\frac{\tau}{2} a\left(q_{1}, \psi\right)+\frac{\tau^{2}}{4} b\left(q_{1}, \psi\right) \\
& =c\left(q_{0}, \psi\right)-\frac{\tau}{2} a\left(q_{0}, \psi\right)-\frac{\tau^{2}}{4} b\left(q_{0}, \psi\right)-\tau b\left(q_{0}, \psi\right)-\frac{\tau^{2}}{4} b\left(\sigma_{0}, \psi\right)+\tau c\left(\rho_{0}, \psi\right) \\
& =\tau c\left(\rho_{0}, \psi\right)-\frac{\tau^{2}}{4} b\left(\sigma_{0}, \psi\right)
\end{aligned}
$$

Now let $\psi=q_{1}$ in the equation above. By using the Cauchy-Schwartz and Young's inequalities and since $a\left(q_{1}, q_{1}\right) \geq 0$,

$$
\begin{aligned}
\left\|q_{1}\right\|_{W}^{2}+\frac{\tau^{2}}{4}\left\|q_{1}\right\|_{V}^{2} & \leq \tau c\left(\rho_{0}, q_{1}\right)-\frac{\tau^{2}}{4} b\left(\sigma_{0}, q_{1}\right) \\
& \leq \tau\left\|\rho_{0}\right\|_{W}\left\|q_{1}\right\|_{W}+\frac{\tau^{2}}{4}\left\|\sigma_{0}\right\|_{V}\left\|q_{1}\right\|_{V} \\
& \leq\left\|q_{1}\right\|_{W}^{2}+\frac{\tau^{2}}{4}\left\|\rho_{0}\right\|_{W}^{2}+\frac{\tau^{2}}{8}\left\|\sigma_{0}\right\|_{V}^{2}+\frac{\tau^{2}}{8}\left\|q_{1}\right\|_{V}^{2} .
\end{aligned}
$$

Using the estimates for $\rho_{0}$ and $\sigma_{0}$ it follows that

$$
\begin{aligned}
\frac{\tau^{2}}{4}\left\|q_{1}\right\|_{V}^{2} & \leq \frac{\tau^{2}}{2}\left\|\rho_{0}\right\|_{W}^{2}+\frac{\tau^{2}}{4}\left\|\sigma_{0}\right\|_{V}^{2} \\
& \leq \frac{\tau^{6}}{2} \max \left\|v_{h}^{\prime \prime \prime}\right\|_{W}^{2}+\frac{\tau^{6}}{4} \max \left\|u_{h}^{\prime \prime \prime}\right\|_{V}^{2}
\end{aligned}
$$

Therefore we obtain the desired estimate:

$$
\begin{aligned}
\left\|e_{1}\right\|_{V}^{2} & \leq \tau^{6} \max \left\|v_{h}^{\prime \prime \prime}\right\|_{W}^{2}+\frac{\tau^{6}}{2} \max \left\|u_{h}^{\prime \prime \prime}\right\|_{V}^{2}+2 \tau^{6} \max \left\|u_{h}^{\prime \prime \prime}\right\|_{V}^{2} \\
& =\tau^{6} \max \left\|v_{h}^{\prime \prime \prime}\right\|_{W}^{2}+\frac{5 \tau^{6}}{2} \max \left\|u_{h}^{\prime \prime \prime}\right\|_{V}^{2} .
\end{aligned}
$$

Substituting the Estimates (6.10) and (6.15) into (6.7) (Lemma 6.1) yields

$$
\begin{aligned}
\max \left\|e_{n}\right\|_{W}^{2} & \leq 40 T^{4} \tau^{4} \max \left\|v_{h}^{\prime \prime \prime}\right\|_{W}^{2}+32 T^{2} \tau^{4} \max \left\|u_{h}^{\prime \prime \prime}\right\|_{W}^{2}+\frac{1}{8} \tau^{6} \max \left\|u_{h}^{\prime \prime \prime}\right\|_{W}^{2} \\
& +\frac{5 \tau^{6}}{16} \max \left\|u_{h}^{\prime \prime \prime}\right\|_{V}^{2}+2 \tau^{8} \max \left\|v_{h}^{\prime \prime \prime}\right\|_{W}^{2}+8 \tau^{6} \max \left\|u_{h}^{\prime \prime \prime}\right\|_{W}^{2} \\
& +2 \tau^{8} K_{a} \max \left\|u_{h}^{\prime \prime \prime}\right\|_{V}^{2} .
\end{aligned}
$$


Note that if $f \in C^{2}([0, T] ; X)$, then $u_{h} \in C^{4}[0, T]$ and consequently the following error estimate is obtained.

Theorem 6.1. If $f \in C^{2}([0, T] ; X)$, then

$$
\begin{aligned}
\left\|u_{h}\left(t_{k}\right)-u_{k}^{h}\right\|_{W} & \leq \tau^{2}\left(7 T^{2} \max \left\|u_{h}^{(4)}\right\|_{W}+3(1+2 T) \max \left\|u_{h}^{\prime \prime \prime}\right\|_{W}\right) \\
& +2 \tau^{3}\left(\max \left\|u_{h}^{(4)}\right\|_{W}+\max \left\|u_{h}^{\prime \prime \prime}\right\|_{V}\right)+\sqrt{2 K_{a}} \tau^{4} \max \left\|u_{h}^{\prime \prime \prime}\right\|_{W}
\end{aligned}
$$

for each $t_{k} \in(0, T)$.

Remark. Estimates for $\left\|u_{h}^{\prime \prime \prime}\right\|_{W},\left\|u_{h}^{(4)}\right\|_{W}$ and $\left\|u_{h}^{\prime \prime \prime}\right\|_{V}$ can be obtained in terms of the initial data and the forcing function.

Finally, error estimates for the fully discrete approximation of the solution of Problem G are obtained by combining results from Section 4 with Theorem 6.1. (Due to Assumption E2, the estimate in Corollary 4.1 translates into an error estimate with respect to $\|\cdot\|_{W}$.)

\section{Applications}

\section{Vibrating systems}

The theory of this article can be applied to any system where elastic bodies interact, provided that the model problem is linear. Numerous examples can be found in [17], for example, networks of linked beams.

A hybrid model where a beam (Euler-Bernoulli) has complex boundary conditions due to a damping tip body attached to one end is considered in [18]. The damping is not of viscous type. It is easy to establish conditions E1 to E4 and hence the theory in this paper can be applied.

Even in relatively simple problems general damping terms may occur. If material (Kelvin-Voigt) damping is present in a tapered Euler-Bernoulli beam, the damping is neither weak nor modal and a convergence theory for general damping is required.

In a recent article [19] the effects of generalized damping distributions on non-homogeneous Timoshenko beams are investigated. The authors mention that so-called proportional damping (or modal damping) is usually modelled 
for simplicity. They stress the importance of other forms of damping, e.g. damping in joints. Damping in joints, like boundary damping, is neither weak nor strong. Clearly, a theory for problems concerning these types of damping is required.

Lastly, consider a plate-beam model in [20]. The model problem referred to as Problem RMT, is a special case of Problem G. Small transverse vibration of a plate is considered, with one beam supporting the plate on part of the boundary. The damping is in the boundary at one endpoint of the beam. It is proved in [20] that Assumptions E1 to E4 are satisfied and the theory in this article is applicable.

\section{The multi-dimensional wave equation}

An obvious application is the multi-dimensional wave equation with boundary damping. (Viscous damping was considered in [2].) Consider the wave equation in a two-dimensional bounded domain denoted by $\Omega$ with boundary $\partial \Omega$. Denote the unit outer normal on the boundary by $n$. Given functions $f, u_{0}$ and $u_{1}$, find $u$ defined on $\Omega \times[0, T]$ such that

$$
\begin{aligned}
\rho \partial_{t}^{2} u & =\nabla \cdot(A \nabla u)+f \text { in } \Omega \times(0, T) \\
u & =0 \text { on } \partial \Omega-\Sigma \\
\partial_{t} u & =-A \nabla u \cdot n \text { on } \Sigma
\end{aligned}
$$

while $u(\cdot, 0)=u_{0}$ and $\partial_{t} u(\cdot, 0)=u_{1}$.

The problem in weak variational form is a special case of Problem G. More detail can be found in [2] where it is proved that Assumptions E1 to E3 are satisfied. We show that E4 hold. Clearly $a$ is symmetric. By the trace theorem [7, Theorem 4.18, p.143] and since $\|\cdot\|_{V}$ is equivalent to the norm of $H^{1}(\Omega)$,

$$
\begin{aligned}
|a(u, v)| & \leq\|\gamma u\|_{\mathcal{L}^{2}(\partial \Omega)}\|\gamma v\|_{\mathcal{L}^{2}(\partial \Omega)} \\
& \leq C\|u\|_{H^{1}(\Omega)}\|v\|_{H^{1}(\Omega)} \leq K_{a}\|u\|_{V}\|v\|_{V} .
\end{aligned}
$$

If $f \in C^{1}\left([0, T] ; \mathcal{L}^{2}(\Omega)\right)$, then there exists a unique solution by Theorem 2.1.

Now consider the finite element approximation of the problem. For simplicity we consider the two-dimensional case and use triangle elements. A finite dimensional subspace $S^{h}$ of $\mathrm{V}$ is constructed, using piecewise linear basis functions.

The Galerkin approximation is a special case of Problem $\mathrm{G}^{h}$. 
Depending on the properties of the boundary of $\Omega$, the parameters, initial values $u_{0}$ and $u_{1}$ and the function $f$, it can be proved that Assumptions A1 and A2 hold. (It is beyond the scope of this article.) For the problem under discussion, we have that $H(V, k)=H^{k}(\Omega) \cap V$. Instead of Assumption A3, we have the following result for piecewise linear basis functions: If $u \in H^{k}(\Omega)$ for $k \geq 2$, then

$$
\|\Pi u-u\|_{V} \leq C_{\Pi} T h|u|_{2}
$$

where $|\cdot|_{2}$ denotes the usual semi-norm.

Note that in [21] the multi-dimensional wave equation with first-order absorbing boundary conditions is treated. It is a more general problem than the example considered above. (In [5] an even more general boundary condition is considered.)

\section{Conclusion}

The examples above clearly demonstrate the applicability of the theory in this article. Application of the results in this article to a given system is in general not trivial. The difficult part is to obtain the necessary estimates.

At present it is not clear how the general theory can be applied to the problem in [5]. However, with some modification, it is a possibility and we consider it a challenge for further research.

\section{Acknowledgements}

The financial assistance of the National Research Foundation of South Africa (NRF) towards this research is hereby acknowledged. Opinions expressed and conclusions arrived at, are those of the authors and are not necessarily to be attributed to the NRF.

The research of the second author was supported by NRF grant 95178. The research of the third author was supported in part by NRF grant IFR2011041700007.

\section{References}

[1] N.F.J. Van Rensburg and A.J. Van Der Merwe (2002). Analysis of the solvability of linear vibration models. Appl. Anal. 81:1143-1159. 
[2] M. Basson and N. F. J. Van Rensburg (2013). Galerkin finite element approximation of general linear second order hyperbolic equations. $\mathrm{Nu}$ mer. Func. Anal. Opt. 34(9):976-1000.

[3] L. Zietsman, A.J. Van Der Merwe and N.F.J. Van Rensburg (1999). Convergence of the FEM solution for the vibration of a damaged beam. Technical report UPWT 99/20. Pretoria: Department of Mathematics and Applied Mathematics, University of Pretoria.

[4] L. Zietsman (2000). Finite element analysis of vibration models with interface conditions, $\mathrm{PhD}$ thesis, University of Pretoria.

[5] T. Dupont (1973). $\mathcal{L}^{2}$-estimates for Galerkin methods for second order hyperbolic equations. SIAM J. Numer. Anal. 10:880-889.

[6] G.A. Baker (1976). Error estimates for finite element methods for second order hyperbolic equations. SIAM J. Numer. Anal. 13:564-576.

[7] J.T. Oden and J.N. Reddy (1976). An introduction to the mathematical theory of finite elements. John Wiley and Sons.

[8] S.R. Wu (2003). A priori error estimates for explicit finite element for linear elasto-dynamics by Galerkin method for central difference method. Comput. Methods Appl. Mech. Engrg. 192:5329-5353.

[9] R.E. Showalter (1977). Hilbert space methods for partial differential equations. Pitman, London.

[10] K. T. Andrews, K. L. Kuttler and M. Shillor (1996). Second order evolution equations with dynamic boundary conditions. J. Math. Anal. Appl. 197:781-795.

[11] J.P. Aubin (1967). Behaviour of the error for the approximate solution of boundary value problems for linear elliptic operators by Galerkin's and finite difference methods. Annali della Scuola Normale di Pisa, Series 3. 21:599-637.

[12] J. Nitsche (1969). Ein Kriterium fur die Quasi-Optimalitat dis ritzchen Verfahrens. Numer. Math. 11:346-348.

[13] M. J. Grote and D. Schötzau (2009). Optimal error estimates for the fully discreet interior penalty discontinuous Galerkin method for the wave equation. J. Sci. Comput. 40:257-272. 
[14] S. Karaa (2011). Error estimates for finite element approximations of a viscous wave equation. J. Sci. Comput. 40:257-272.

[15] S. Karaa (2011). Finite element $\theta$-schemes for the acoustic wave equation. Adv. Appl. Math. Mech. 3:181-203.

[16] S. Karaa (2012). Stability and convergence of fully discrete finite element schemes for the acoustic wave equation. J. Appl. Math. Comput. 40:659682.

[17] J.E. Lagnese, G. Leugering and E.J.P.G. Schmidt(1994). Modeling Analysis and Control of Dynamic Elastic Multi-Link Structures. Birkhäuser, Boston.

[18] K.T. Andrews and M. Shillor (2002). Vibrations of a beam with a damping tip body. Math. Comput. Modeling. 35:1033-1042.

[19] S. Sorrentino, A. Fasana and S. Marchesiello (2007). Analysis of nonhomogeneous Timoshenko beams with generalized damping distributions. J. Sound Vibration 304:779-792.

[20] N.F.J. Van Rensburg, L. Zietsman and A.J. Van Der Merwe (2009). Solvability of a Reissner-Mindlin-Timoshenko plate-beam vibration model. IMA J. Appl. Math. 74:149-162.

[21] L.C. Cowsar, T.F. Dupont and M.F. Wheeler (1996). A priori estimates for mixed finite element approximations of second-order hyperbolic equations with absorbing boundary conditions.. SIAM J. Numer. Anal. 33(2):492-504. 